\title{
A Purified Serum Glycopeptide from Controls and Cystic Fibrosis Patients. I. Comparison of their Mucociliary Activity on Rabbit Tracheal Explants
}

\author{
MIRIAM G. BLITZER ${ }^{(36)}$ AND EMMANUEL SHAPIRA ${ }^{(38)}$ \\ Hayward Genetics Center, Departments of Pediatrics and Pathology, Tulane University School of Medicine, \\ New Orleans, Louisiana, USA
}

\begin{abstract}
Summary
A glycopeptide with a molecular weight of approximately $\mathbf{5 0 0 0}$ was purified from sera of controls and cystic fibrosis patients. Both the cystic fibrosis and control glycopeptides revealed the absence of aromatic amino acids. The effect of the purified glycopeptide on rabbit tracheal explants was examined in a doubleblind fashion. The purified fraction from cystic fibrosis patients caused marked ciliary dyskinetic activity and profound mucus secretion. The equivalent fraction from controls, even in a 50-fold higher concentration, showed no apparent biological activity on rabbit tracheal explants.
\end{abstract}

\section{Speculation}

A glycopeptide was purified from sera of controls and cystic fibrosis patients. The glycopeptide from cystic fibrosis patients revealed marked mucociliary activity, whereas its "normal counterpart" demonstrated no such activity. Abnormalities in some aspects of glycoprotein metabolism as a possible underlying defect in cystic fibrosis have been previously suggested. Thus, the mucociliary activity of the cystic fibrosis glycopeptide might be part of a generalized phenomenon of abnormal synthesis or degradation of glycoproteins and glycopeptides.

The basic defect in cystic fibrosis (CF), the most common significant autosomal recessive disorder affecting Caucasians, remains as yet unknown. Abnormalities in exocrine gland secretion, proteolytic enzymes and their inhibitors, polyamine metabolism and the presence of factors presumably unique to CF have been described and reviewed $(17,22)$. Abnormalities in some aspects of glycoprotein metabolism as the possible underlying defect in $\mathrm{CF}$ have been suggested $(1,4,23,29)$.

The presence of a CF-specific factor affecting the rhythmic beat of cilia from rabbit tracheal explants was first described in 1967 by Spock et al. (31). These authors documented this biologic activity (ciliary dyskinesia) in both CF sera and the euglobulin fraction of CF obligate heterozygotes, but not from most normal controls. This factor or factors was observed to disrupt the ciliary rhythm in other ciliated organisms, including oyster gill cilia (10), fresh water mussels (6) and flagellated bacteria (14). Media from leukocyte, fibroblast and amniotic fluid cell cultures of $\mathrm{CF}$ patients also revealed the presence of a factor or factors with ciliary dyskinetic activity $(5,11,15)$. The ciliary dyskinetic factor from sera of CF patients has been partially purified and characterized $(9,12,34,35)$. It was shown to be associated with the immunoglobulin $G$ fraction $(3,9,12,34)$ and was dissociated in the presence of $8 \mathrm{M}$ urea (34) or $6 \mathrm{~N}$ guanidine (9). This protein fraction has been shown to have molecular weight ranges between $6000-11,000(9)$ and 3500-10,000 (34).

In the present study, a glycopeptide fraction was purified from sera of controls and CF patients using a similar methodology as previously suggested for the purification of the CF-protein (34). The mucociliary activity of the purified glycopeptide on rabbit tracheal explants was studied.

\section{MATERIALS AND METHODS}

Materials. Bio-Gel P-10, 50-150 mesh, and diethylaminoethyl (DEAE)-cellulose (Cellex-D) were obtained from Bio-Rad Laboratories (Richmond, CA). Silica Gel $60 \mathrm{~F}-254$ precoated thin layer chromatographic plates were from EM Reagents (MCEM Manufacturing, Cincinnati, $\mathrm{OH}$ ). Agarose-EF and ampholines, $\mathrm{pH} 3.5$ 10, for isoelectric focusing were obtained from LKB Instruments, Inc. (Rockville, MD). Bovine serum albumin (BSA) and ribonuclease-B were from Sigma Chemical Company (St. Louis, MO). Regular insulin was purchased from Eli Lilly and Co. (Indianapolis, IN). Diaflo membranes, PM-10 and UM-2, were from Amicon Corporation (Lexington, MA). Dulbecco's Modified Eagles Medium was from GIBCO (Grand Island, NY). Rabbit antisera to human serum proteins and to human immunoglobulin $G$ and goat antiserum to rabbit immunoglobulin $\mathrm{G}$ were from Calbiochem-Behring Corporation (La Jolla, CA). All other reagents were either reagent grade or the best grade available.

Blood specimens. Blood specimens (10-120 ml) were obtained, following signed, informed consent. Control specimens were obtained from four healthy individuals with no family history of CF ( 2 males, 22 and 28 years of age, and 2 females, 14 and 25 years of age). Specimens from ten CF patients, six males and four females in the age range of 2-25 years, were obtained. The clinical diagnosis of $\mathrm{CF}$ was confirmed by repeated sweat electrolyte analyses (sweat chloride: $70-105 \mathrm{meq} / \mathrm{liter}$ ). All patients are followed and managed by the Louisiana CF Clinic. At the time when blood specimens were obtained, nine patients were receiving supplementary pancreatic enzymes (Cotazym or Pancrease) and eight patients were receiving antibiotics (oxacillin, bactrim, tetracycline, etc.). The blood was incubated for $30 \mathrm{~min}$ at $37^{\circ} \mathrm{C}$, the clot separated and retracted for $3 \mathrm{~h}$ at $4^{\circ} \mathrm{C}$. The serum was separated by centrifugation and stored at $-20^{\circ} \mathrm{C}$ until use.

Ciliary dyskinesia bioassay. The sera, immunoglobulin $\mathrm{G}$ fractions and purified glycopeptides were examined for their effect on rabbit tracheal explants as described by Conover et al. (16), using Dulbecco's Modified Eagles Medium containing 15\% fetal calf serum supplemented with L-glutamine, sodium pyruvate, antibiotics and fungizone. Explants were examined for viability using a Nikon inverted microscope. Following 2-3 days of incubation $\left(37^{\circ} \mathrm{C}\right.$ in $5 \% \mathrm{CO}_{2}$ atmosphere), explants with synchronously beating cilia were chosen for the assay. Explants were placed on a glass slide, excess media was removed and three drops of the examined preparation (serum, immunoglobulin $\mathrm{G}$ preparation or the purified glycopeptide at $37^{\circ} \mathrm{C}$ ) were added. Ciliary movement was examined with a Reichert phase microscope and followed for at least $45 \mathrm{~min}$ at room temperature (approximately $25^{\circ} \mathrm{C}$ ). Photomicrographs were obtained using an American Optical microscope. 
Purification of the glycopeptide. The glycopeptide was purified from four individual control sera, one individual $\mathrm{CF}$ serum and two pooled CF sera. Each pooled CF specimen was from four different affected patients in order to account for the possible heterogeneity of the disease entity. All control sera were negative for ciliary dyskinetic activity, whereas all CF specimens were positive. To $35 \mathrm{ml}$ of serum, ethylenediaminetetraacetic acid (EDTA) was added to a final concentration of $10 \mathrm{mM}$, stirred for $30 \mathrm{~min}$, and dialyzed against a 200 -fold excess of $0.01 \mathrm{M}$ potassium phosphate buffer, $\mathrm{pH} 8.0$, containing $2 \mathrm{mM}$ EDTA. The dialyzed serum was applied to a DEAE-cellulose column $(25 \mathrm{~cm} \times 3 \mathrm{~cm})$, preequilibrated and eluted with the same phosphate-EDTA buffer. All column chromatography was run at $4^{\circ} \mathrm{C}$. To the unadsorbed fraction (mainly immunoglobulin $\mathrm{G}$ by immunoelectrophoresis), solid urea was added to a final concentration of $8 \mathrm{M}$ and stirred for $1 \mathrm{~h}$ at $37^{\circ} \mathrm{C}$. Components with molecular weights of less than 10,000 were collected by ultrafiltration through an Amicon Diaflo Membrane PM-10. This fraction was concentrated to $5 \mathrm{ml}$ using an Amicon Diaflo Membrane UM-2 (molecular weight cut-off 1000). After concentration, the volume was reconstituted to $180 \mathrm{ml}$ with distilled water and reconcentrated to $5 \mathrm{ml}$. Most of the urea and other low molecular weight components were removed by this procedure. The concentrated fraction was applied to a Bio-Gel P-10 column $(103 \mathrm{~cm} \times 2.4 \mathrm{~cm})$, preequilibrated and eluted with $0.05 \mathrm{M}$ ammonium bicarbonate, $\mathrm{pH} 7.8$. The eluted fractions were monitored as to their absorbancy at 220 $\mathrm{nm}$. The column was precalibrated using a mixture of BSA, ribonuclease-B and insulin (5 mg each) as molecular weight markers. One major peak with an elution volume similar to that of insulin was found in both the control and CF samples. This peak was pooled, lyophilized, and dissolved in $4 \mathrm{ml}$ distilled water. The purification yield of the various preparations was $1.2-1.8 \mathrm{mg}$ / $35 \mathrm{ml}$ of control serum and $1.5-2.1 \mathrm{mg} / 35 \mathrm{ml}$ of $\mathrm{CF}$ serum (determined by Lowry).

Thin layer chromatography. Ascending thin layer chromatography of the glycopeptide was performed on silica gel plates $(0.2$ $\mathrm{mm}$, preheated at $120^{\circ} \mathrm{C}$ prior to use) and developed in $n$-pro$\mathrm{panol} / \mathrm{acetic}$ acid/water (3:2:2). Samples were applied in triplicate sets. After drying, one set was stained with ninhydrin $(1.0 \mathrm{~g}$ ninhydrin, $0.1 \mathrm{~g}$ cadmium acetate, $10 \mathrm{ml}$ distilled water and 100 $\mathrm{ml}$ acetone), one set with orcinol (1\% in 50\% sulfuric acid) and one set with resorcinol $(0.1 \%$ resorcinol, $0.125 \mathrm{mM}$ copper sulfate in $4.8 \mathrm{~N}$ hydrochloric acid). Bands were visualized after heating at $120^{\circ} \mathrm{C}$.

Analytical procedures. Protein content was determined according to Lowry et al. (20) using BSA as reference solutions. Protein concentration was also calculated from the ultraviolet (UV) absorption spectra by measuring the relative absorbancy at $225 \mathrm{~nm}$ and $215 \mathrm{~nm}$ according to the procedure of Murphy and Kies (21).

Isoelectric focusing was performed using $0.8 \%$ agarose gels containing 2\% ampholines ( $\mathrm{pH} 3.5-10)$ using an LKB Multiphor 2117 system, according to the manufacturer's instructions. Focusing was complete within $90-120$ min using constant power $(6.25$ watts per gel). The $\mathrm{pH}$ gradient was determined with a surface $\mathrm{pH}$ electrode (LKB 2117-111 Multiphor $\mathrm{pH}$ electrode). The gel was immediately fixed, dried and stained with Periodic Acid-Schiff stain. Following periodic oxidation ( $1 \mathrm{~h}$ in $1 \%$ periodic acid in $3 \%$ acetic acid), the gels were rinsed with distilled water and stained for $1 \mathrm{~h}$ in Schiff stain $(0.67 \%$ basic fuchsin, $0.1 \mathrm{M}$ sodium metabisulfite in $15 \%$ hydrochloric acid).

Sialic acid content was determined by the modified thiobarbituric acid procedure (30).

Spectrophotometric measurements were made on a Beckman DB spectrophotometer (Model 24) with silica cells with a $1 \mathrm{~cm}$ light path. UV absorption spectra were obtained in ammonium bicarbonate, $\mathrm{pH} 7.8$, in the wavelength range of $200-300 \mathrm{~nm}$.

Immunological procedures. Double gel diffusion and immunoelectrophoresis were performed as previously described (27). In order to increase the sensitivity of the procedures, a second antibody technique was used. After exhaustive washing, goat antiserum to rabbit immunoglobulin was applied in the wells (double gel diffusion) or troughs (immunoelectrophoresis). The plates were incubated for another $24 \mathrm{~h}$ at room temperature, washed exhaustively in phophate buffered saline $(0.14 \mathrm{M}$ sodium chloride in $0.01 \mathrm{M}$ phosphate buffer, $\mathrm{pH} \mathrm{7.0;} \mathrm{PBS)} \mathrm{and} \mathrm{the}$ immune precipitate stained with $1 \%$ amido black.

\section{RESULTS}

Physical and biochemical characterization of the purified fractions. The purified fractions were obtained as a single homogeneous peak by Bio-Gel P-10 chromatography. The fraction obtained from CF patients had a somewhat larger elution volume than the fraction purified from controls. The results are shown in Figure 1. The apparent molecular weights, as calculated for the eluted fractions (Fig. 2), were 5700 for controls and 4600 for the sample from CF patients. An aliquot of the purified, lyophilized sample was dissolved in PBS and rechromatographed on a Bio-Gel P-10 column $(59 \mathrm{~cm} \times 1 \mathrm{~cm})$, preequilibrated and eluted with PBS. In PBS, both the control and CF purified fractions formed dimers with apparent molecular weights of 8700 for $C F$ and 11,200 for controls (Fig. 2).

The purified preparations from controls and CF patients were examined as to their UV absorption spectra and compared to that of BSA. An identical absorption spectrum was revealed for the purified preparations from controls and CF patients that differed markedly from that of BSA. The UV spectra of all purified preparations were characterized by the absence of tryptophan and tyrosine absorbancies. No absorbancy in the wavelength range of $300-260 \mathrm{~nm}$ was observed with the purified glycopeptides. A single peak, with maximum absorbancy at $206 \mathrm{~nm}$ in ammonium bicarbonate $(0.05 \mathrm{M}, \mathrm{pH} 7.8)$ was found with all purified preparations. When the protein content of the glycopeptides was calculated from the UV spectrum, it was found to be close to 8-fold higher than that determined according to Lowry; with BSA, identical values were found by both procedures.

The purified fractions from controls and CF patients $(14 \mu \mathrm{g}$ protein according to Lowry) were compared by thin layer chromatography. Only one band, positive with ninhydrin and orcinol and negative with resorcinol, was revealed. The results are shown in Figure 3. These results indicated the homogeneity and glycopeptide nature of the purified fractions. The CF purified preparation had a somewhat higher chromatographic mobility when compared to the controls. The absence of sialic acid in the purified glycopeptide, as suggested by the negative resorcinol staining, was

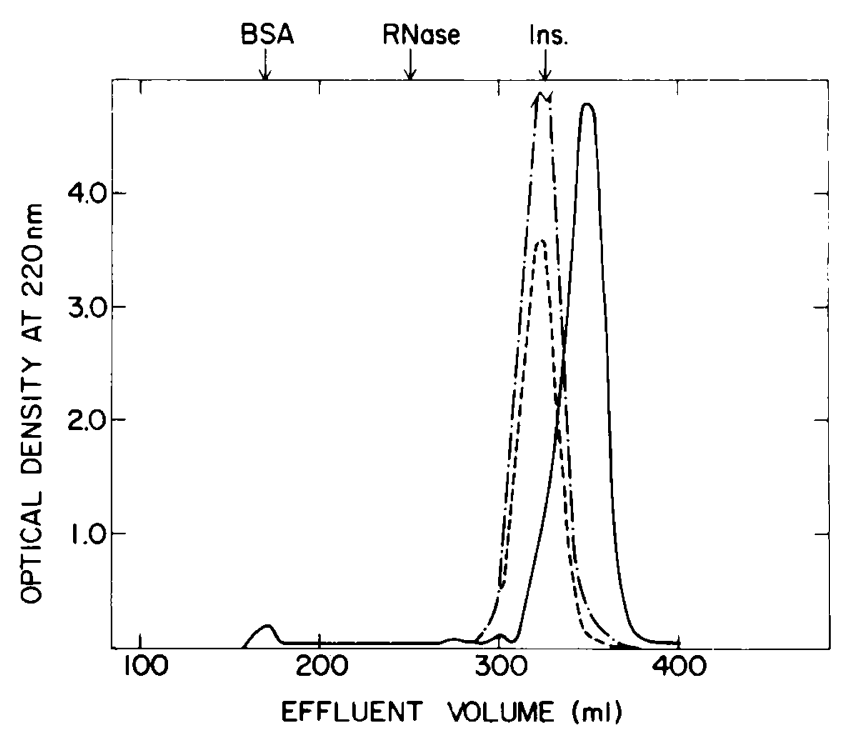

Fig. 1. Bio-Gel P-10 column chromatography $(103 \mathrm{~cm} \times 2.4 \mathrm{~cm})$ profiles of two preparations from controls (broken line) and one from a CF patient (solid line). The arrows indicate the elution volumes of bovine serum albumin (BSA), bovine ribonuclease-B (RNase) and bovine insulin (Ins). 


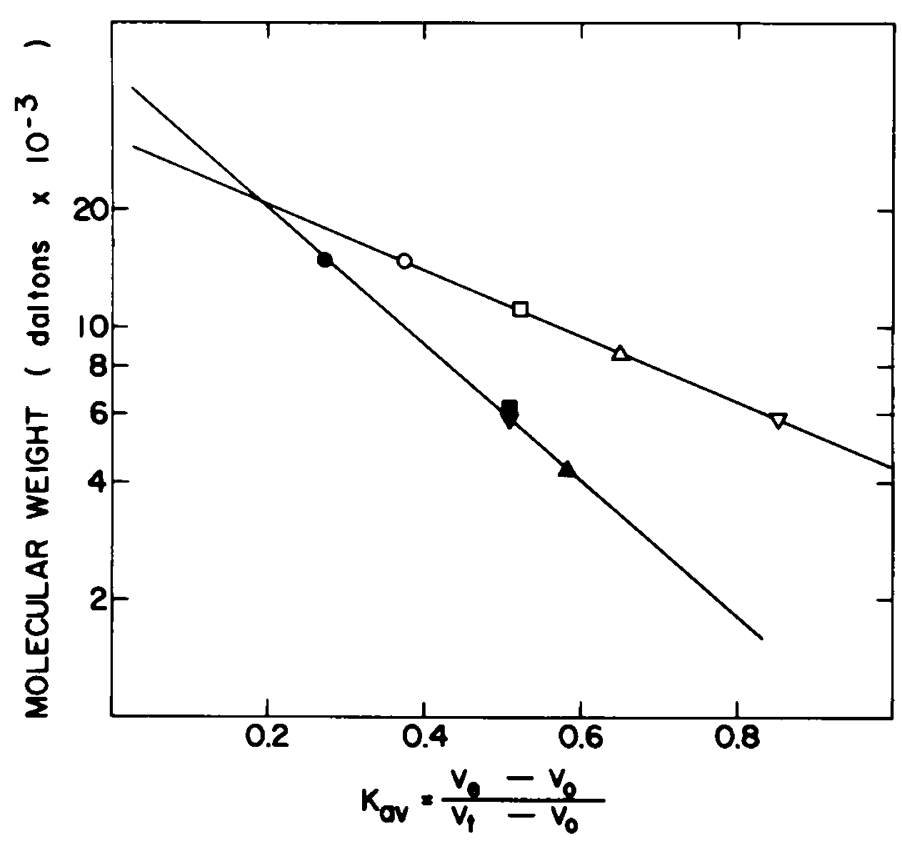

Fig. 2. Molecular weights of the purified glycopeptides as estimated by Bio-Gel P-10 chromatography (controls, $\square, \square$; CF patients, $\Delta, \Delta$ ). The reference proteins are ribonuclease-B $(O, \Theta)$ and bovine insulin $(\nabla, \nabla)$.

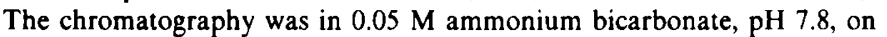
a $103 \mathrm{~cm} \times 2.4 \mathrm{~cm}$ column (solid symbols, light line) and rechromatographed on a $59 \mathrm{~cm} \times 1 \mathrm{~cm}$ column in phosphate buffered saline (open symbols, heavy line).

further documented using a more sensitive, quantitative assay, the modified thiobarbituric acid procedure (30). No sialic acid was detected in the acid hydrolysate, $0.35 \mathrm{mg} / \mathrm{ml}$ in $1 \mathrm{~N}$ sulfuric acid.

The purity of the glycopeptide preparations was further documented when the preparations were compared by isoelectric focusing. The three preparations from CF patients and two from controls, $40 \mu \mathrm{g}$ each, were applied and stained with Periodic AcidSchiff stain for carbohydrates. Only one band (isoelectric point approximately 4.8 ), identical for both the CF and the control glycopeptide preparations, was revealed.

The purified glycopeptide preparations from CF and controls, $0.4 \mathrm{mg} / \mathrm{ml}$ by Lowry, were examined by double gel diffusion and immunoelectrophoresis against antiserum to human serum proteins and antiserum to human immunoglobulin G. No immune precipitate bands were revealed with either antisera, using both procedures. The absence of any immune precipitating, cross-reacting material with these antisera was further documented by using the second antibody technique, with goat antiserum to rabbit immunoglobulin $\mathrm{G}$.

Comparison of control and CF purified glycopeptides by the ciliary dyskinesia bioassay. The results of the experiments thus far described established the glycopeptide nature of the fractions purified from both controls and CF patients. The purified glycopeptide was then diluted with PBS, pH 7.0, and the ciliary dyskinetic activity of these fractions was compared. The samples were coded in a double-blind fashion and examined by each of the authors individually. The three preparations from the CF patients revealed ciliary dyskinetic activity in concentrations as low as $1 \mu \mathrm{g} / \mathrm{ml}$, whereas the four preparations from controls did not alter the ciliary rhythm, even in concentrations as high as $50 \mu \mathrm{g} / \mathrm{ml}$ (concentrations determined by Lowry). With the purified preparations, an obvious distinction between a positive and negative ciliary dyskinetic reaction was observed, whereas with the individual serum samples, the distinction was less certain. In the presence of the purified glycopeptide from controls, the tracheal explants maintained normal cilia movement and appearance for at least 60 min, indistinguishable from that seen in the culture medium. The glycopeptide purified from CF patients $(10 \mu \mathrm{g} / \mathrm{ml})$ caused ciliary dyskinesia within 30 seconds, and gradually, swelling and exces- sive secretion and accumulation of mucus-like droplets were observed. The results are shown in Figure 4. The purified glycopeptide from controls, in the same concentration $(10 \mu \mathrm{g} / \mathrm{ml})$, did not alter the rhythmic movement of the cilia and did not change its physical appearance (bottom of Figure 4). When an area with a mucus gland was examined in the presence of the CF glycopeptide $(10 \mu \mathrm{g} / \mathrm{ml})$, the appearance of a "volcano" to some observers or a "roman candle" to others was seen (Fig. 5).

\section{DISCUSSION}

The ciliary dyskinetic factor observed originally by Spock et al. (31) in sera of CF patients was later partially purified and characterized by others $(9,12,34,35)$. These investigators $(8,34,35)$ suggested that this factor was a peptide, molecular weight between 3500 and 11,000 , and a specific marker for the CF gene (32). In her recent review article, Dr. Bowman (8) raised the question of whether or not there is a normal counterpart to the $\mathrm{CF}$ factor. In the present study, using a similar purification procedure as previously suggested by Wilson and Fudenberg (34), a glycopeptide purified from CF serum had marked mucociliary dyskinetic activity (Fig. 4), whereas its "normal counterpart" revealed no such activity (Fig. 4). This glycopeptide was purified in similar yield from both CF and control sera, and only the biological activity of this fraction was found to be CF-specific. The glycopeptides purified from controls and CF patients had molecular weights in the same range as previously suggested for the CF factors, 6000 11,000 for the "CF factor" (9) and 3500-10,000 for the "CF protein" (34). In low ionic strength ( $0.05 \mathrm{M}$ ammonium bicarbonate, $\mathrm{pH} 7.8$ ), the apparent molecular weight was 4600 for the $\mathrm{CF}$ glycopeptide and 5700 for controls, whereas in higher molarity (PBS, pH 7.0), a dimer with molecular weight 11,200 for controls and 8700 for CF was observed (Fig. 2). This phenomenon of

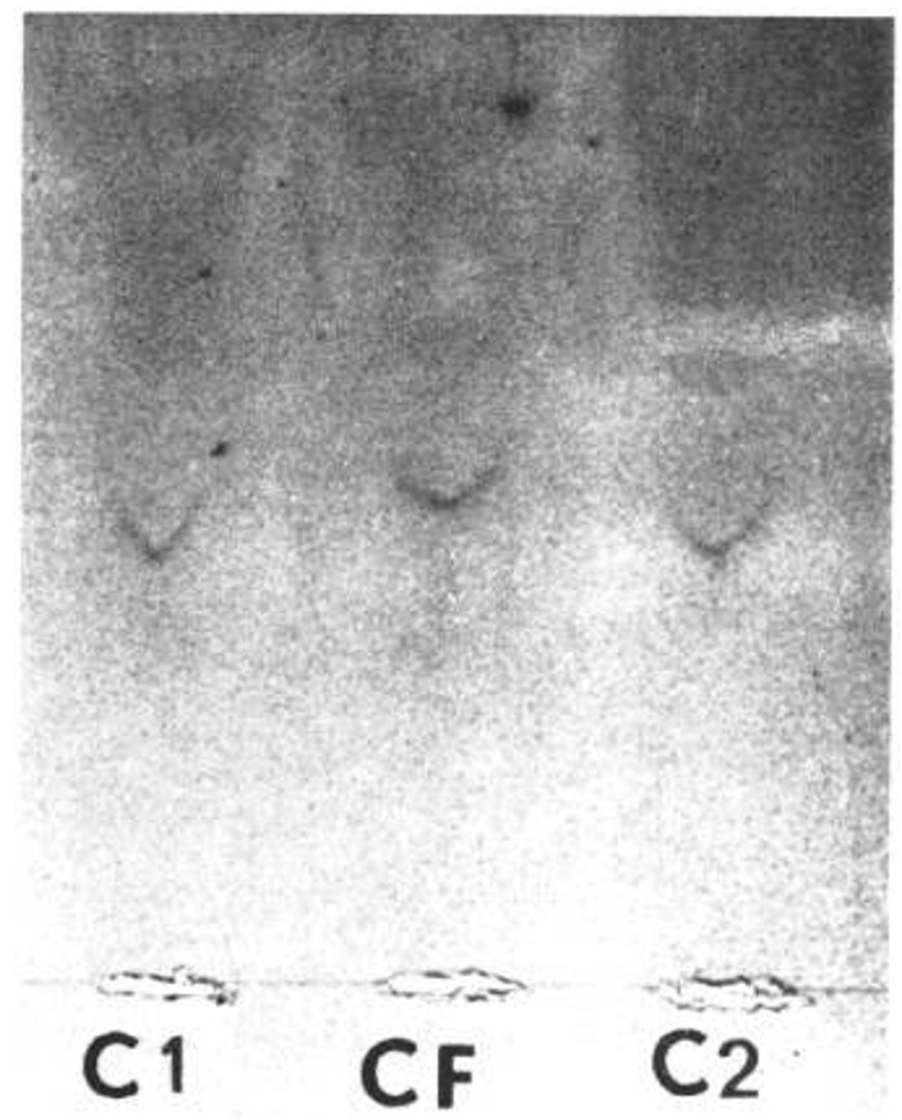

Fig. 3. Thin layer chromatography of the purified glycopeptides from sera of controls ( $\mathrm{Cl}$ and $\mathrm{C} 2$ ) and from cystic fibrosis patients (CF). The single band in each preparation in the figure was revealed by orcinol staining. 

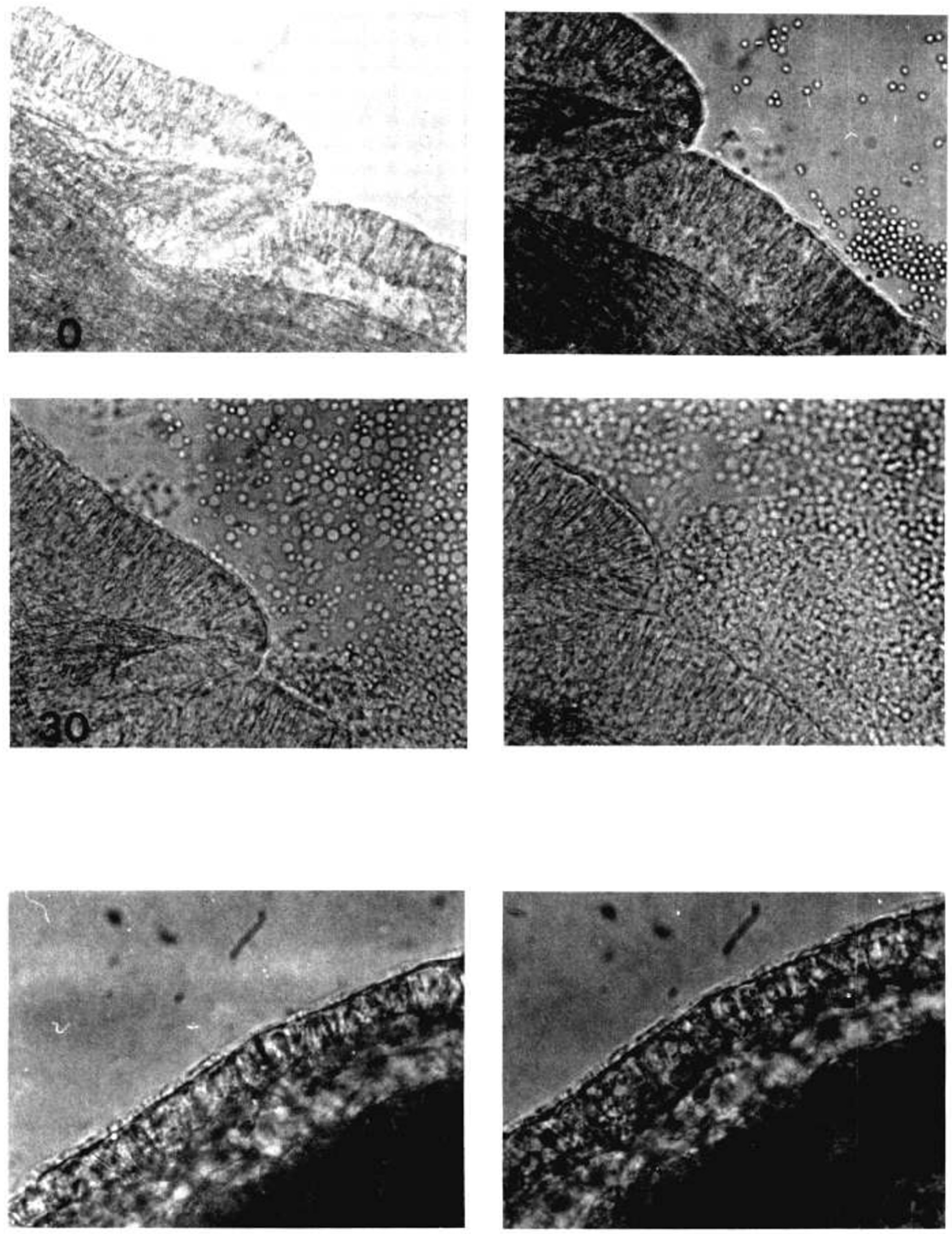

Fig. 4. Photomicrographs of rabbit tracheal explants $(\times 520)$ in the presence of $10 \mu \mathrm{g} / \mathrm{ml}$ of the purified glycopeptides. In the presence of the cystic fibrosis glycopeptide (upper panels), note the disappearance of the ciliary border immediately following application (0) and the gradual appearance of edema and accumulation of mucus-like droplets at 15,30 and $45 \mathrm{~min}(15,30,45)$. In the presence of the preparation from controls (lower panels). note the distinct ciliary border immediately after application (0) and no change after $45 \mathrm{~min}(45)$. 


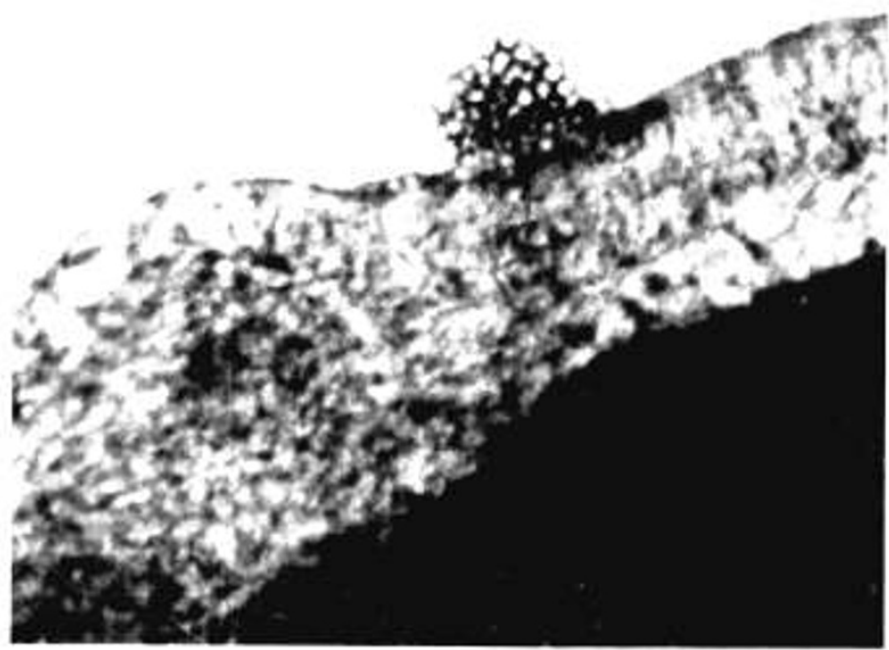

Fig. 5. Photomicrograph of rabbit tracheal explant mucus gland $7 \mathrm{~min}$ following application of purified glycopeptide $(10 \mu \mathrm{g} / \mathrm{ml})$ from cystic fibrosis patients. Note the marked accumulation of mucus at the orifice.

dimer formation might explain the relatively broad range of molecular weights previously observed for the CF factors or CF protein.

This highly purified glycopeptide (Fig. 3), from both control and CF specimens, had a UV absorption spectrum that was characterized by the absence of tyrosine and tryptophan absorbancies. The absence of aromatic amino acids was further documented by the discrepancy between the protein concentration as calculated from the UV absorbancy (21) and that determined by the Lowry et al. method (20). The chromogenic product of the Lowry procedure is influenced by the presence of aromatic amino acids, amino acid sequence and peptide bonds, whereas the UV difference between $225 \mathrm{~nm}$ and $215 \mathrm{~nm}$ is not affected by the presence of aromatic amino acids $(13,21)$. The purified glycopeptide from both $\mathrm{CF}$ and controls was positive with orcinol staining and negative with resorcinol staining. The deficiency of sialic acid in the carbohydrate moiety of the glycopeptide was further demonstrated using the thiobarbituric acid assay (30).

Both rabbit antiserum to human serum proteins and rabbit antiserum to human immunoglobulin $G$ did not reveal any precipitating antibodies toward the purified glycopeptide. These observations might indicate that this glycopeptide is a poor immunogen. At present, we are comparing the immunologic properties of the purified glycopeptide from controls and CF patients using monoclonal antibodies. Preliminary results indicate that the glycopeptides from controls and $\mathrm{CF}$ patients have most of their antigenic determinants in common. Using this methodology, a larger number of controls, patients, and patient controls (patients with chronic lung disease other than $\mathrm{CF}$ ) can be studied in order to document the reproducibility and significance of these findings.

In the present study, a similar glycopeptide was purified from controls and CF patients using the same purification methodology. The UV absorption spectra of both were indistinguishable, the molecular weights were very similar as were the chromatographic patterns. These observations indicate the structural similarities between the CF glycopeptide and its "normal counterpart", which differ significantly in their biologic activity toward rabbit tracheal explants. The purified CF glycopeptide revealed marked mucociliary activity whereas its normal counterpart had no apparent effect on these explants (Fig. 4 and 5).

Abnormalities in the glycoprotein composition of duodenal fluid (18), salivary secretions (19) and tracheobronchial secretions (7) have previously been described in CF patients. More recently, functional $(25,26,28)$ and structural $(4,33)$ alterations in alpha- 2-macroglobulin, a serum glycoprotein, were documented. Increased binding of the lectin concanavalin $A$ to alpha-2-macroglobulin as well as to immunoglobulin $G$ and to immunoglobulin $\mathbf{M}$ from CF patients was also observed (29). Alhadeff et al. (2) documented an altered carbohydrate moiety of the lysosomal enzyme alpha-fucosidase from CF patients. Differences of monosaccharide composition of membrane glycopeptides from CF and control fibroblasts were described by Scanlin and Glick (24). Thus, abnormalities in some aspects of glycoprotein metabolism were suggested as a possible underlying defect in $C F(1,4,23,29)$. The observation in the present study of mucociliary activity by the CF glycopeptide, shown to be absent in its "normal counterpart", might be a consequence of such a generalized phenomenon of abnormal synthesis or degradation of glycoproteins and glycopeptides.

\section{REFERENCES AND NOTES}

1. Alhadeff, J. A.: Glycoproteins and cystic fibrosis: A review. Clin. Genet. 14: 189 (1978).

2. Alhadeff, J. A.. Watkins, P.. and Freeze, H.: Purification and characterization of altered cystic fibrosis liver $\alpha$-L-fucosidase. Clin. Genet. 13: 417 (1978).

3. Barnett. D. R., Schanfield, M. S., McCombs, M. L., and Bowman. B. H.: Isoelectric focusing and IgG allotyping of the serum fraction containing cystic fibrosis ciliary inhibitor. Tex. Rep. Biol. Med., 31: 709 (1973).

4. Ben-Yoseph, Y.. DeFranco, C. L., and Nadler, H. L.: Decreased sialic acid and altered binding to lectins of purified $\alpha_{2}$-macroglobulin from patients with cystic fibrosis. Clin. Chim. Acta, 99: 31 (1979).

5. Beratis, N. G., Conover, J. H., Conod, E. J., Bonforte, R. J., and Hirschhorn, K. Studies on ciliary dyskinesia factor in cystic fibrosis. III. Skin tibroblasts and cultured amniotic fluid cells. Pediatr. Res., 7: 958 (1973).

6. Besley, G. T. N., Patrick, A. D., and Norman, A. P.: Inhibition of the motility of gill cilia of Dreissenia by plasma of cystic fibrosis patients and their parents. $J$ Med. Genet., 6: 278 (1969).

7. Boat, T. F., Cheng, P. W., Iyer, R. N., Carlson, D. M., and Polony, I.: Human respiratory tract secretions: mucous glycoproteins of nonpurulent tracheobronchial secretions and sputum of patients with bronchitis and cystic fibrosis. Arch. Biochem. Biophys., 177: 95 (1976).

8. Bowman, B. H.: Cystic fibrosis: Review of investigation of the mucociliary inhibitor. Tex. Rep. Biol. Med., $38: 47$ (1979).

9. Bowman, B. H., Lankford, B. J., Fuller, G. M., Carson, S. D., Kurosky, A., and Barnett, D. R.: Cystic fibrosis: The ciliary inhibitor is a small polypeptide associated with immunoglobulin G. Biochem Biophys. Res. Commun., 64 : 1310 (1975).

10. Bowman. B. H., Lockhart, L. H., and McCombs, M. L.: Oyster ciliary inhibition by cystic fibrosis factor. Science, 164: 325 (1969).

11. Bowman, B. H., Lockhart, L. H., Herzberg. V. L.. Barnett. D. R., and Kramer. J.: Cystic fibrosis: Synthesis of ciliary inhibitor by amniotic cells. Clin. Genet 4: 461 (1973)

12. Bowman, B. H., McCombs, M. L., and Lockhart, L. H.: Cystic fibrosis: Characterization of the inhibitor to ciliary action in oyster gills. Science, 167: 871 (1970).

13. Chou, S. -C. and Goldstein, A.: Chromogenic groupings in the Lowry protein determination. Biochem. J., 75: 109 (1960).

14. Cohen, F. L. and Daniel, W. L.: Effects of cystic fibrosis sera on Proteus vulgaris motility. J. Med. Genet., 1I: 253 (1974).

15. Conover, J. H., Beratis, N. G., Conod, E. J., Ainbender, E., and Hirschhorn, K. Studies on ciliary dyskinesia factor in cystic fibrosis. II. Short term leukocyte cultures and long term lymphoid lines. Pediatr. Res., 7: 224 (1973).

16. Conover, J. H.. Bonforte, R. J., Hathaway, P., Paciug, S., Conod, E. J., Hirschhorn, K., and Kopel, K. B.: Studies on ciliary dyskinesia factor in cystic fibrosis. I. Bioassay and heterozygote detection in serum. Pediatr. Res., 7: 220 (1973).

17. di Sant'Agnese. P. A. and Davis, P. B.: Research in cystic fibrosis. N. Engl. J. Med., 295: 481, 534, 597 (1976).

18. Dische. Z.. Pallavicini, C.. Cizek, L. J.. and Chien. S.: Changes in the control of secretion of mucus glycoproteins as possible pathogenic factor in cystic fibrosis of the pancreas. Ann. N.Y. Acad. Sci., 93: 526 (1962).

19. Kutty, K. M., Chandra, R. K., Way, R. C., and Ittycheria, S.: Glycoproteins in the saliva and platelets of cystic fibrosis patients. Cystic Fibrosis Club Abstracts. 17th Annual Meeting. April 1976, St. Louis, MO. p. 45.

20. Lowry, O. H., Rosebrough, N. J., Farr, A. L., and Randall, R. J.: Protein measurement with the Folin phenol reagent. J. Biol. Chem., 193: 265 (1951).

21. Murphy, J. B. and Kies, M. W.: Note on spectrophotometric determination of proteins in dilute solutions. Biochem. Biophys. Acta. 45:382 (1960).

22. Nadler, H. L., Rao, G. J. S., and Taussig, L. M.: Cystic fibrosis. In: J. B. Stanbury, J. B. Wyngaarden, and D. S. Fredrickson: The Metabolic Basis of Inherited Disease. p. 1683 (McGraw-Hill Book Co., New York, 1978).

23. Pearson, R. D. and Lubin, A. H.: Increased heparin binding in cystic fibrosis: A reflection of altered glycoprotein biosynthesis? Pediatr. Res., 13: 834 (1979).

24. Scanlin, T. F. and Glick, M. C.: Differences in monosaccharide composition of membrane glycopeptides from cystic fibrosis and control skin fibroblasts. Pediatr. Res.. 11: 463 (Abstract) (1977).

25. Shapira, E., Ben-Yoseph, Y., and Nadler, H. L.: Decreased formation of $\alpha_{2^{-}}$ 
macroglobulin-protease complexes in plasma of patients with cystic fibrosis. Biochem. Biophys. Res. Commun., 71: 864 (1976).

26. Shapira, E., Ben-Yoseph, Y., and Nadler, H. L.: Abnormal breakdown of $\alpha_{2}-$ macroglobulin-trypsin complex in cystic fibrosis. Clin. Chim. Acta, 78: 359 (1977).

27. Shapira, E., DeGregorio, R., and Nadler, H. L.: Immunologic studies of arylsulfatase $\mathrm{A}$ in normal and metachromatic leukodystrophy liver. Pediatr. Res., I2: 199 (1978).

28. Shapira, E., Martin, A. L., and Nadler, H. L.: Comparison between purified $\alpha_{2-}$ macroglobulin preparations from normal controls and patients with cystic fibrosis. J. Biol. Chem., 252: 7923 (1977).

29. Shapira, E. and Menendez, R.: Increased binding of concanavalin A to $\alpha_{2-}$ macroglobulin. IgM and IgG from cystic fibrosis plasma. Biochem. Biophys. Res. Commun., 93: 50 (1980).

30. Skorza, L. and Mohos, S.: Stable thiobarbituric acid chromophore with dimethyl sulphoxide. Biochem. J.. 159: 457 (1976).

31. Spock, A., Heick, H. M. C., Cress, H., and Logan, W. S.: Abnormal serum factor in patients with cystic fibrosis of the pancreas. Pediatr. Res., 1: 173 (1967).

32. Wilson, G. B.: Cystic fibrosis protein, a confirmed diagnostic marker for detecting heterozygote carriers: Significance in relation to future screening and to a proposed defect in alpha-2-macroglobulin. Pediatr. Res., 13: 1079 (1979).
33. Wilson, G. B. and Fudenberg, H. H.: Studies on cystic fibrosis using isoelectric focusing. II. Demonstration of deficient proteolytic cleavage of $\alpha_{2}$-macroglobulin in cystic fibrosis plasma. Pediatr. Res., 10: 87 (1976).

34. Wilson. G. B. and Fudenberg. H. H.: Further purification and characterization of serum proteins used to detect cystic fibrosis genotypes by isoelectric focusing. Tex. Rep. Biol. Med., 34: 51 (1976).

35. Wilson, G. B., Monsher, M. T., and Fudenberg. H. H.: Studies on cystic fibrosis using isoelectric focusing. III. Correlation between cystic fibrosis protein and ciliary dyskinesia activity in serum shown by a modified rabbit tracheal bioassay. Pediatr. Res., 11: 143 (1977).

36. This study was performed in partial fulfillment of requirements for Ph.D. thesis

37. The authors thank Dr. R. C. Beckerman for providing us with the CF blood specimens: Dr. P. D. Walker for the photomicrographs, and Dr. Y. T. Li for his comments and suggestions.

38. Requests for reprints should be addressed to: Dr. Emmanuel Shapira. Head. Sections of Clinical and Biochemical Genetics, Department of Pediatrics. Tulane University School of Medicine, 1430 Tulane Avenue. New Orleans, LA 70112.

39. This research was supported by a grant from the Cystic Fibrosis Foundation

40. Received for publication February 18, 1981.

41. Accepted for publication September 23, 1981. 